\title{
CARPAS PARA AUDITORIO EN PINAR DEL REY. MADRID/ESPAÑA
}

\author{
(TENTS FOR THE PINAR DEL REY AUDITORIUM - MADRID/SPAIN)
}

José Miguel de Prada Poole, Dr. Arquitecto

$146-99$

\section{RESUMEN}

Se describe, en este artículo, los materiales y proceso de montaje empleados para construir con una estructura modular tensada, la cubierta del escenario de un auditorio cubierto dado el interés que ofrece lo inédito de su tecnología y la posibilidad de experimentar las cualidades espaciales de la misma en estos casos.

A su vez, al exponer los criterios que llevaron a su empleo, se ponen de manifiesto las ventajas económicas y de montaje, frente a sus implicitas dificultades de proyecto:

- superficies de doble curvatura anticlástica;

- deformación no lineal de la estructura;

- ajuste de empalmes y piezas;

- diseño y fabricación de piezas especiales.

\section{SUMMARY}

The setting up process and the materials used in the construction of the stage covering of an open auditorium, with a tauten modular structure, are described in view of the interest that offers the newness of its technology and the possibility to experiment its spacial qualities in this cases.

Moreover, the criteria that decided its use are explained, and there are showed its economic advantages and its easy setting, taking into account the implicit difficulties of the project:

- double curved anticlastic suface

- non-linear deformation of the structure

- fitting of parts and joints

- design and manufacture of special parts

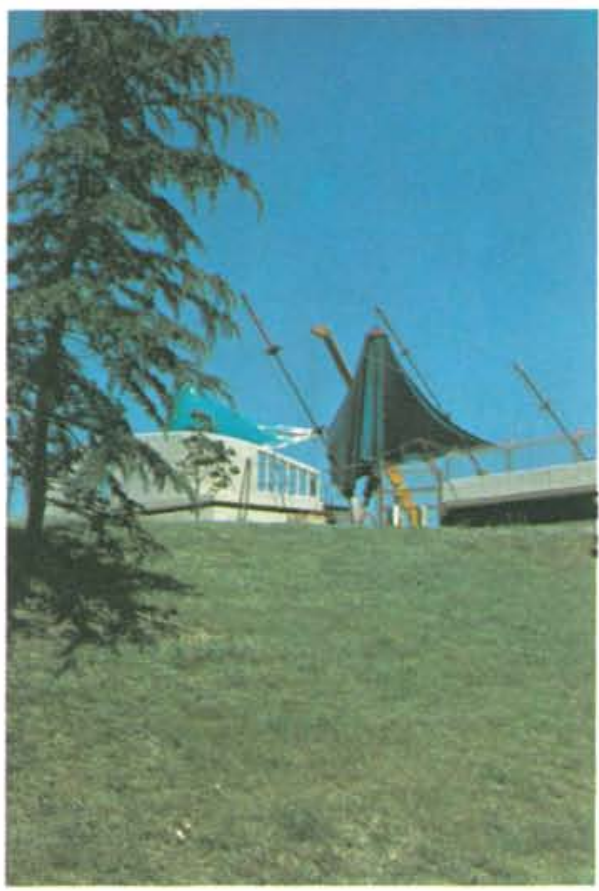

Con esta estructura tensada se cubren el escenario y aledaños del auditorio situado en el parque del Pinar del Rey (Hortaleza) proyectado y promovido por el Ayuntamiento de Madrid. Su propósito principal es crear un umbráculo impermeable sobre la pista, a la vez que delimitar el campo de visión y contar con un hito urbano en el parque.

Se eligió este tipo de estructura por motivos a la vez económicos y de velocidad de montaje, dada la necesidad de contar con el recinto acondicionado en unas fechas determinadas.

- Las carpas cubren una superficie de: 625 m² $^{2}$ salvando unas luces libres de $30 \mathrm{~m}$, con alturas bajo techo entre $3 \mathrm{~m}$ y $12 \mathrm{~m}$;

- son de lona plastificada de poliéster tratada;

- el cableado es de acero protegido con galvanizado;

- las piezas mecánicas, de enganche y articulación, son de acero inoxidable;

- los mástiles, también de acero, apoyan sobre una cimentación de hormigón armado calculados para soportar unas cargas a compresión de $7 \mathrm{t}$ a $20 \mathrm{t}$;

- su peso es de $1.800 \mathrm{~kg}\left(3 \mathrm{~kg} / \mathrm{m}^{2}\right)$;

- las piezas de sus componentes se fabricaron en dos meses; su montaje duró 28 dias con una cuadrilla de tres hombres no especialistas y una grúa de pluma telescópica sobre camión. 

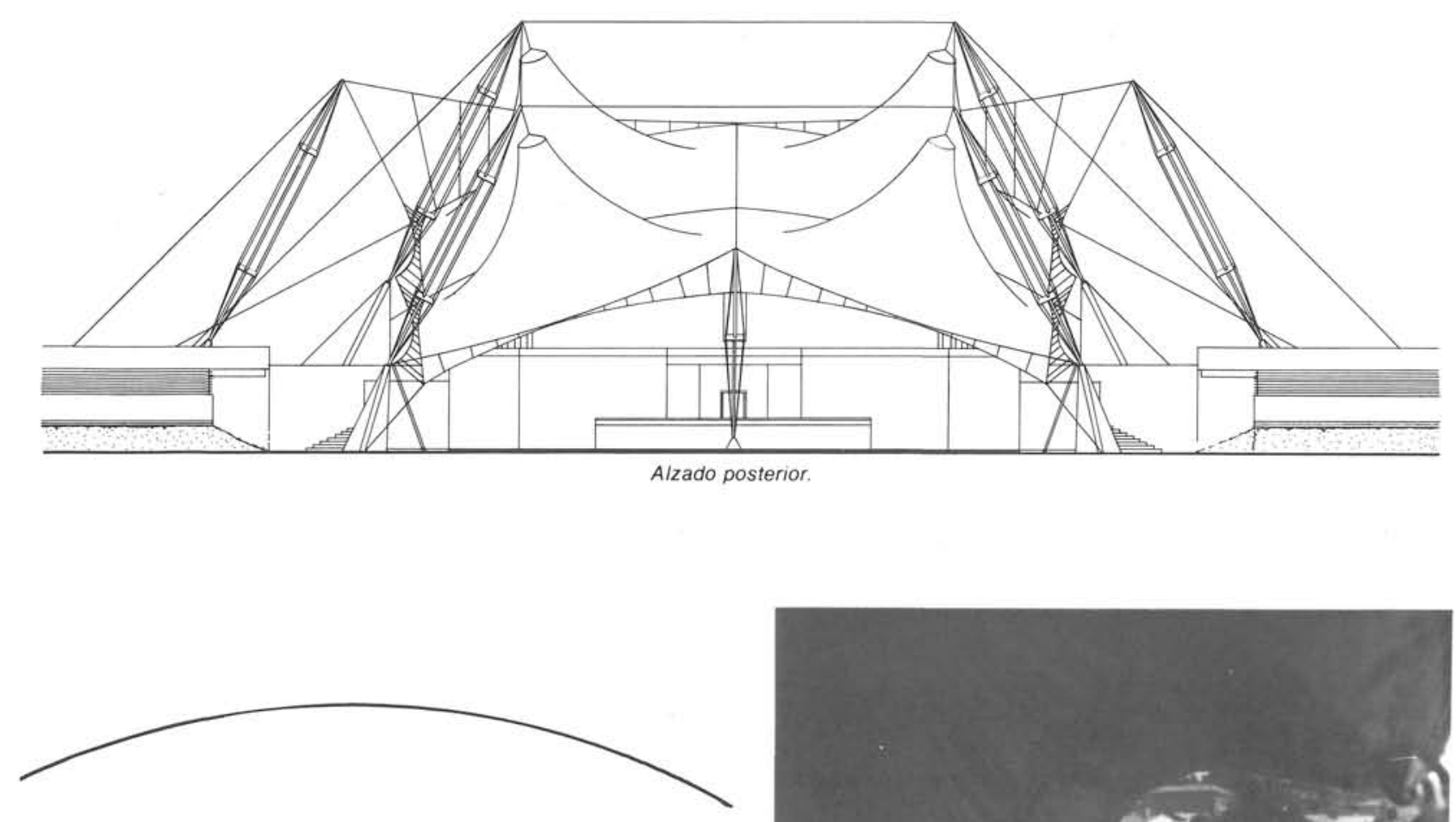

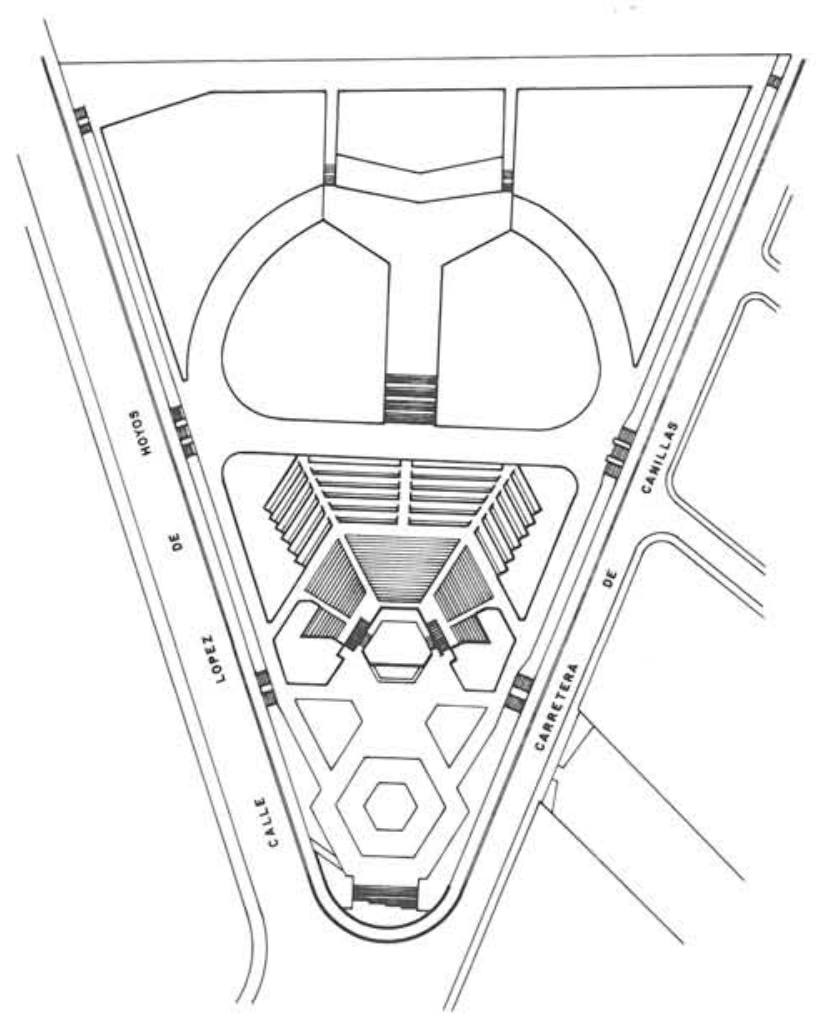

Situacion en el parque.

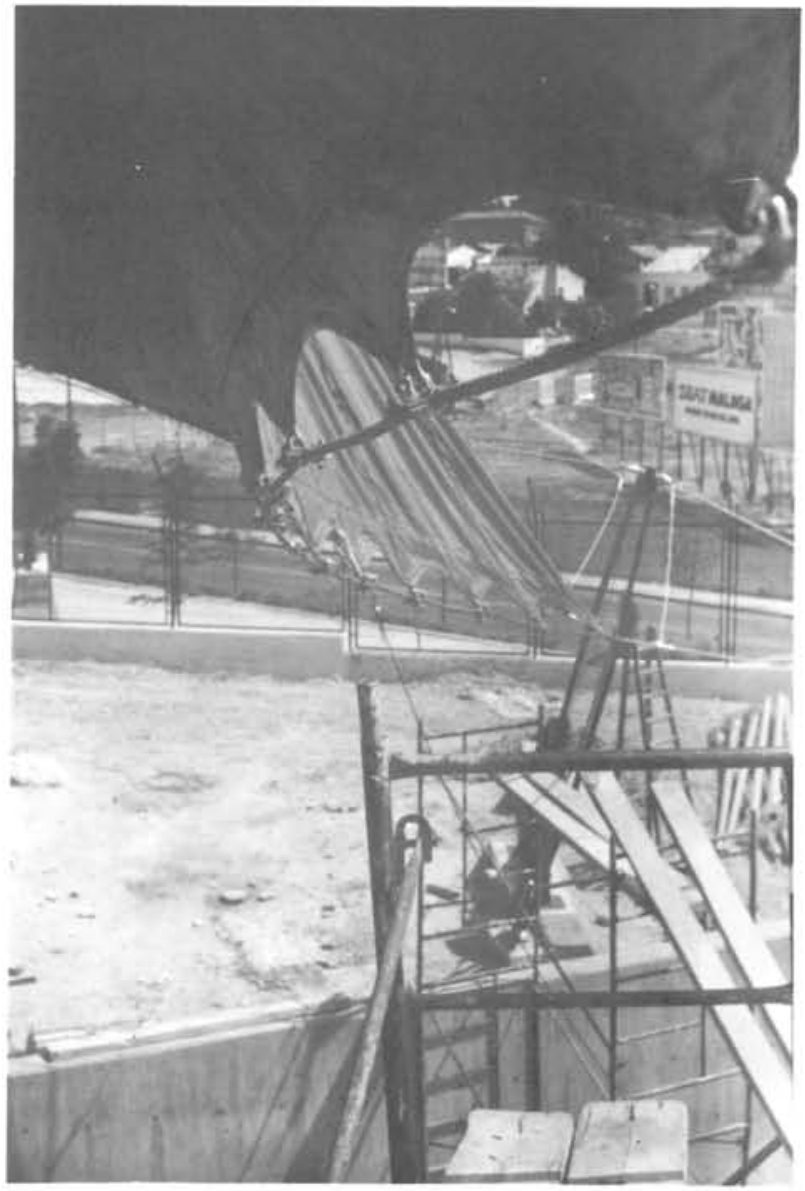

Fig. 1.-Fijacion de cables coaxiles.

Sin embargo, frente a las ventajas que presentan estas membranas cara a su eficacia estructural y constructiva (rapidez de montaje y economia de medios) el proyecto de las mismas plantea serias dificultades a medida que crece su dimensión (superficies alabeadas, dimensiones reales en proyecciones oblicuas; la resistencia al viento crece con el cuadrado de sus dimensiones; más y mejores condiciones de seguridad). 


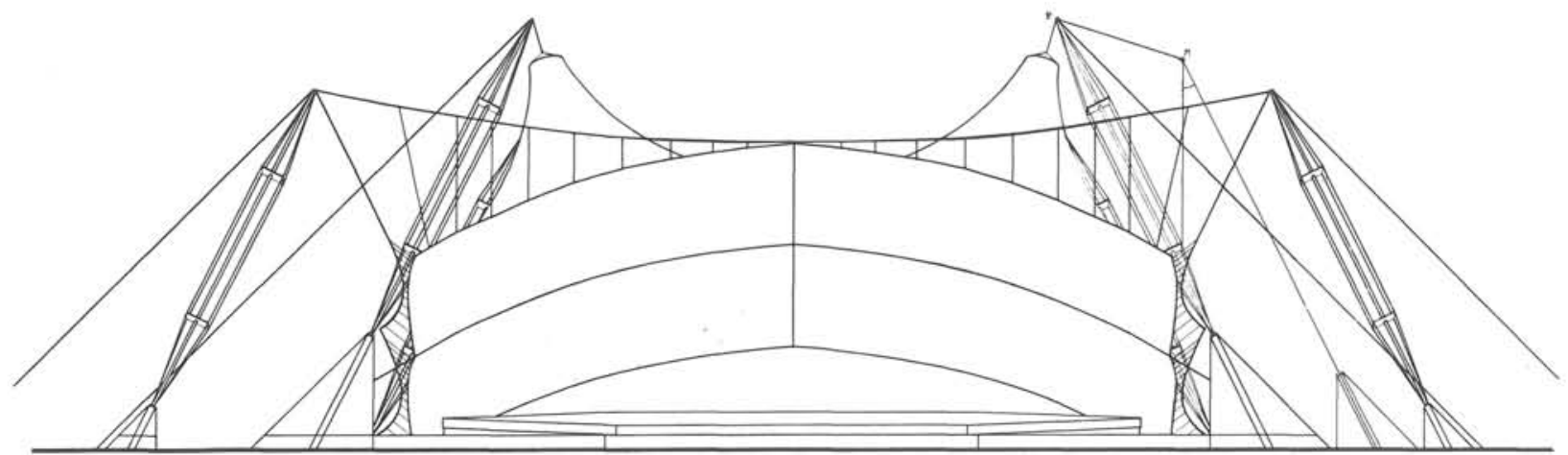

Alzado frontal

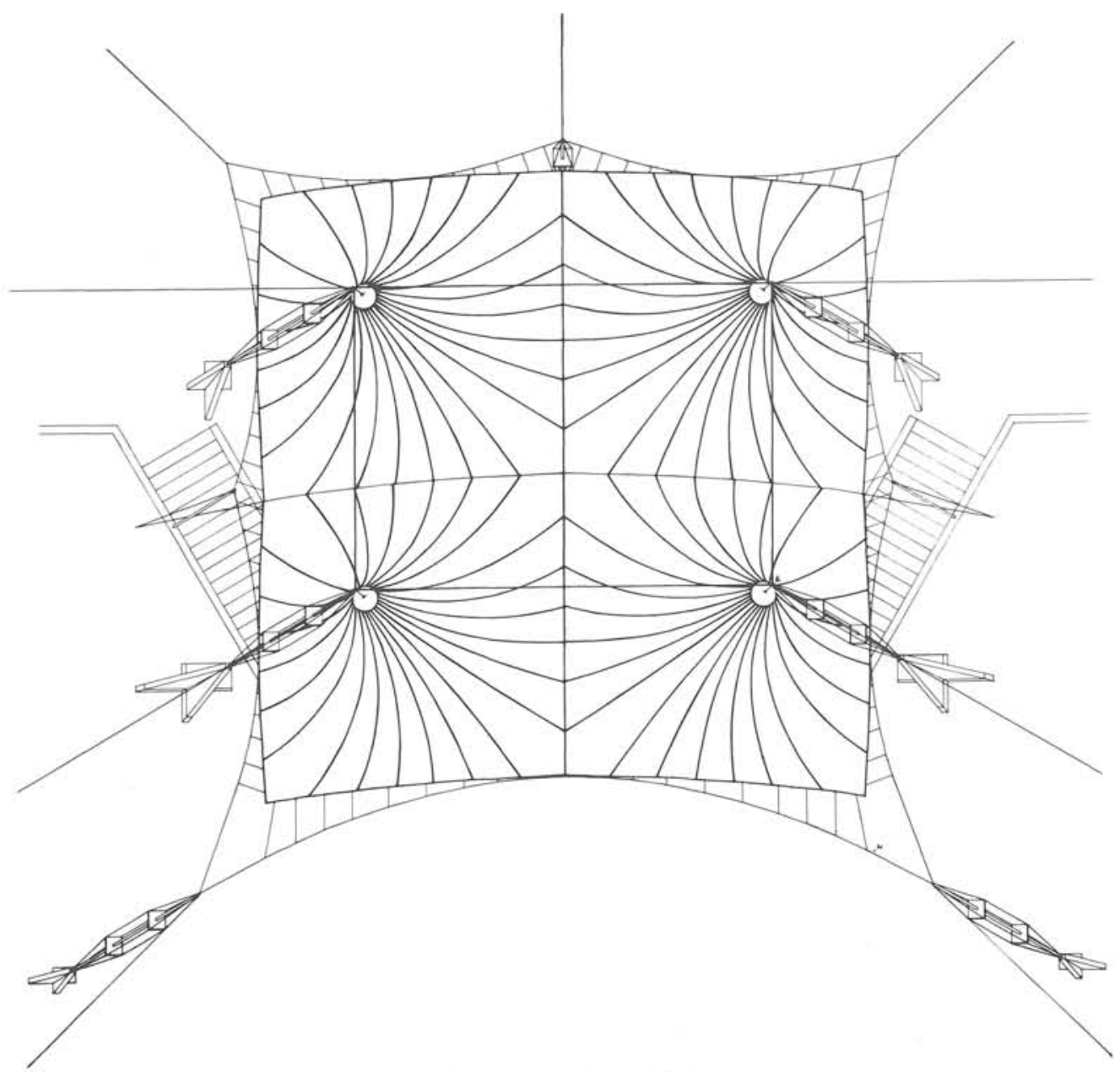

Planta general de cubierta.
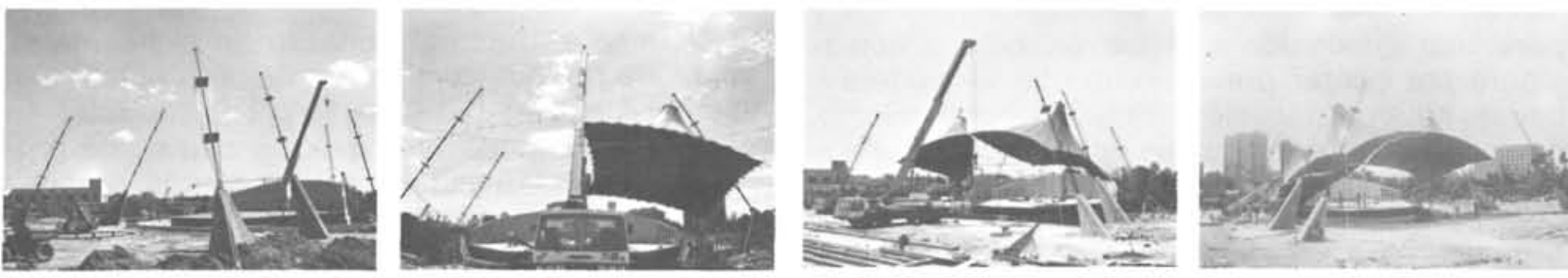

Proceso de montaje. 

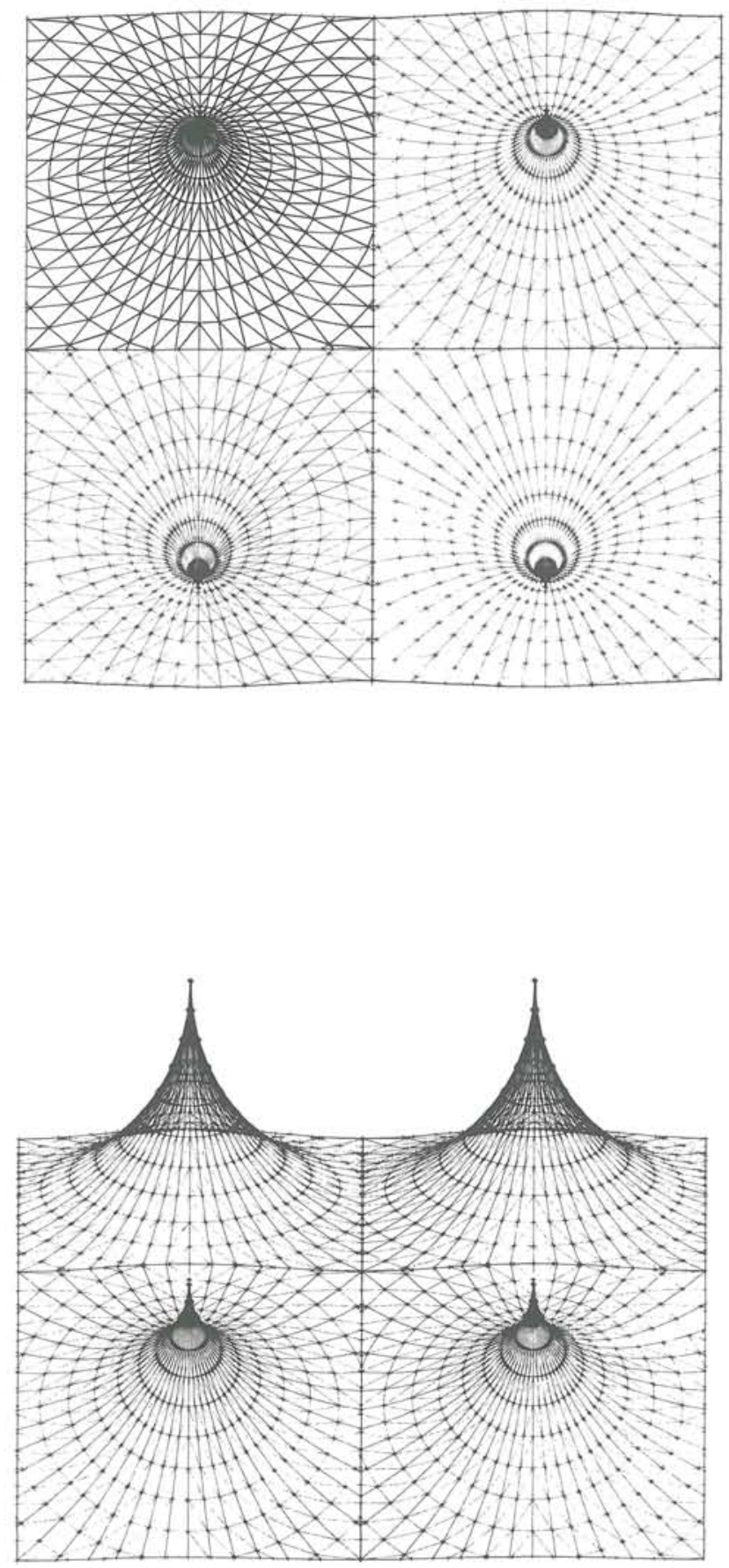

La superficie de la estructura, en si, está formada por cuatro módulos iguales, generado cada uno como superficie de revolución a partir de un arco de circunferencia. No fue proyectada, en principio, para ser colocada en esta situación y con estas dimensiones, sino con el plano de sus casquetes superiores en posición horizontal y para una dimensión aún mayor; pero precisamente por contar previamente con el modelo y poder hacer aplicación inmediata del mismo, cumpliendo las condiciones económicas de velocidad, luces disponibles, tipo de apoyos que emplea para adaptarse a la edificación existente, hizo que esta solución fuese preferible frente a otras (Fig. a, pág. 44).
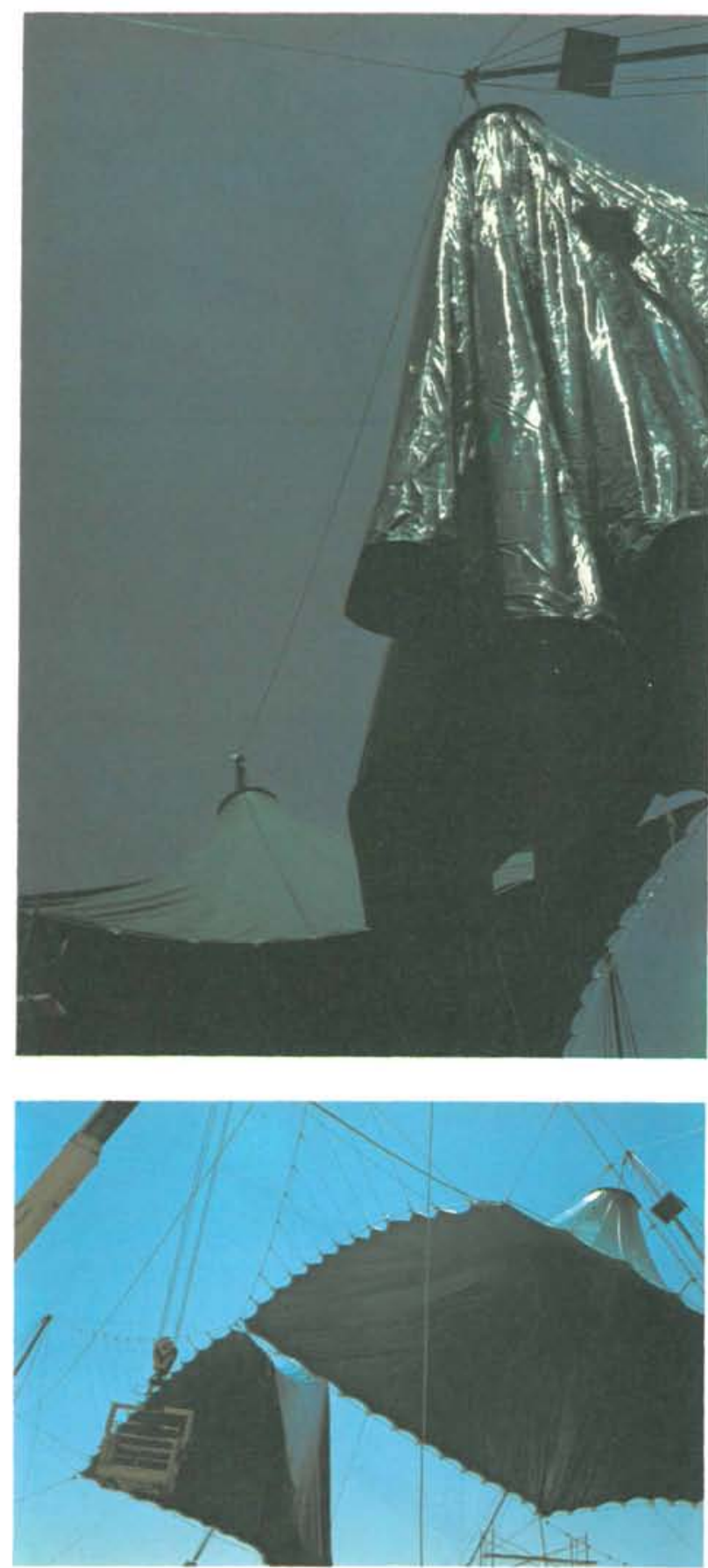

Fig. 2.-Arco a traccion alabeado.

La curvatura de las cuatro superficies fueron corregidas mediante ordenador para hacer compatibles sus bordes (sus caracteristicas geometricas) con la forma de trabajo, que no admite puntos de inflexión.

El sistema estructural utilizado en el perimetro, "cuelgue del borde" a otra catenaria paralela al mismo, fue utilizado para forzar y controlar su curvatura evitando, asi, que los cables de prolongación en las esquinas lleve en sus anclajes a unas distancias incómodas para las condiciones de entorno creadas por el parque. Esto lleva consigo el cálculo de unos arcos de desarrollo 


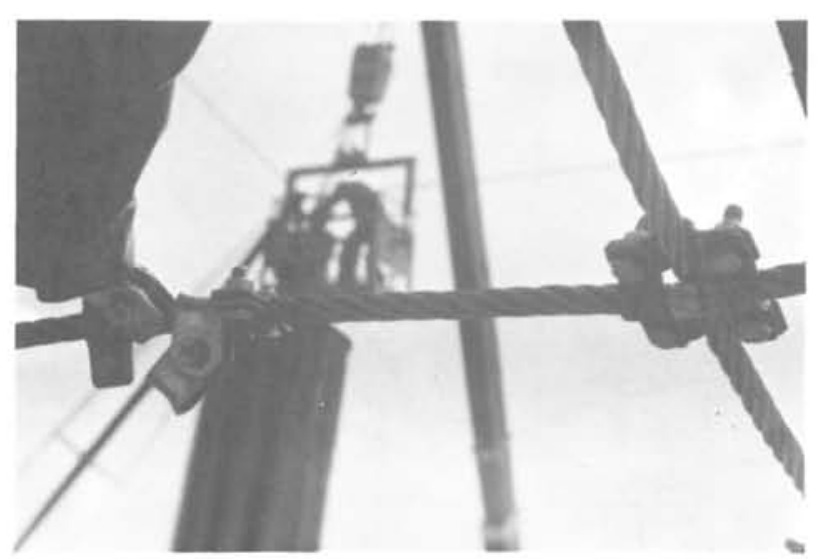

Pieza central en el cruce de cables y pieza de conexion al cable de borde.

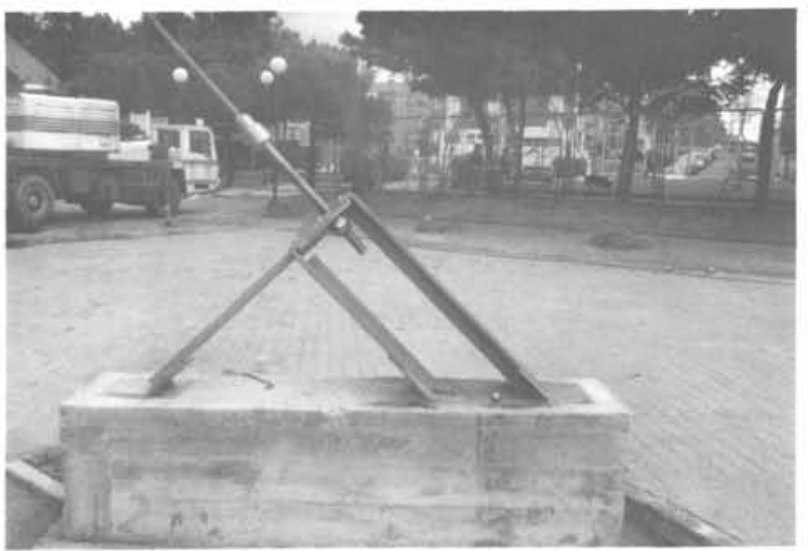

Extremo de cables con tensor.

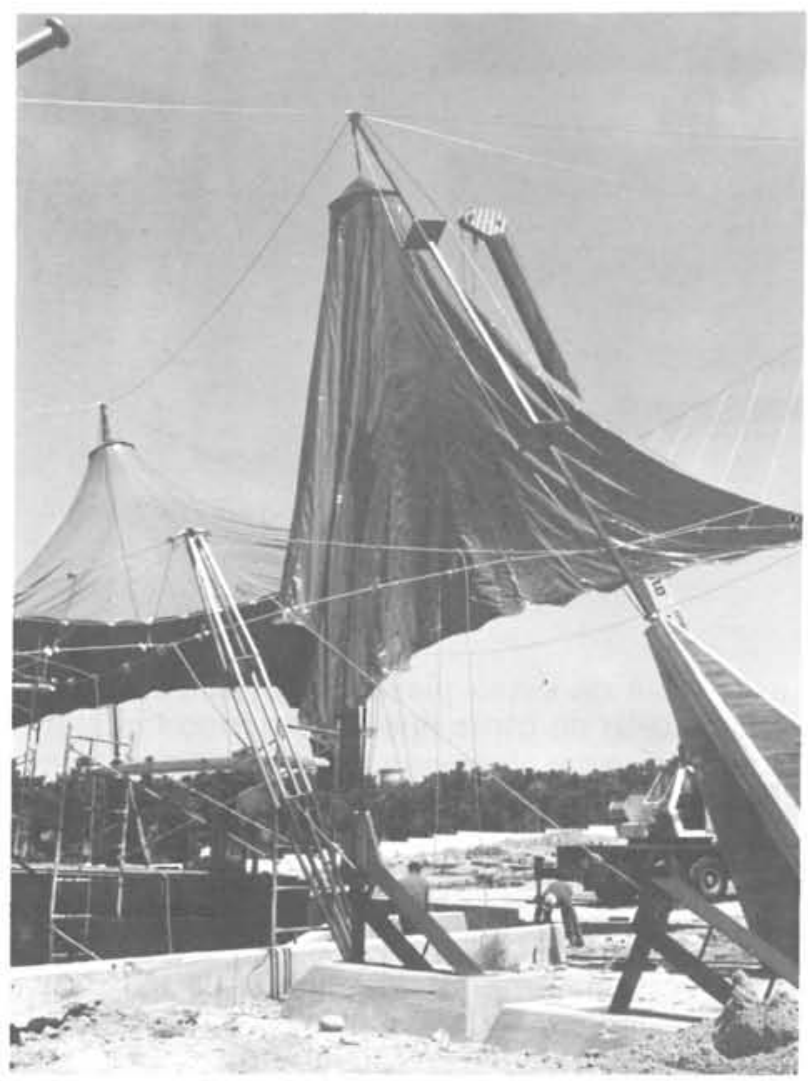

Cableado coaxil en el borde y apoyo de anclaje a cimentación.

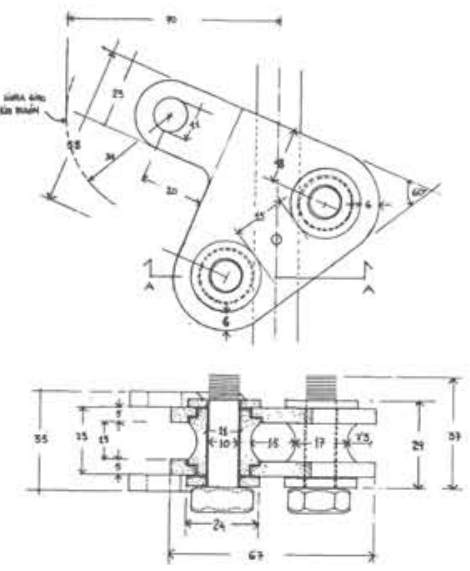

Pieza A de conexion de los estribos del arco a su borde.

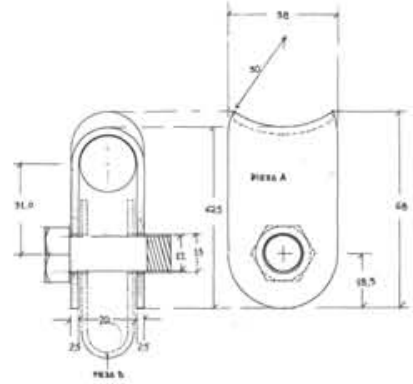

Pieza de conexion a cable de borde.

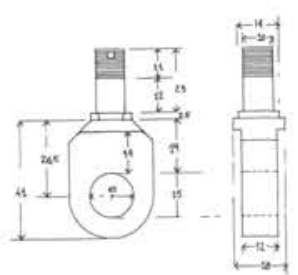

Terminal de estribo a pieza $A$.

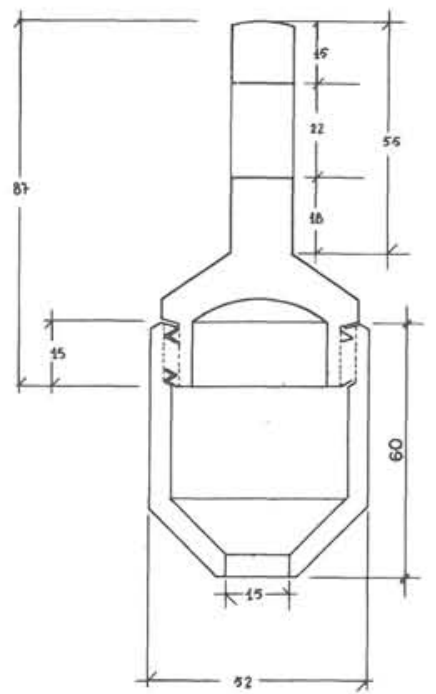

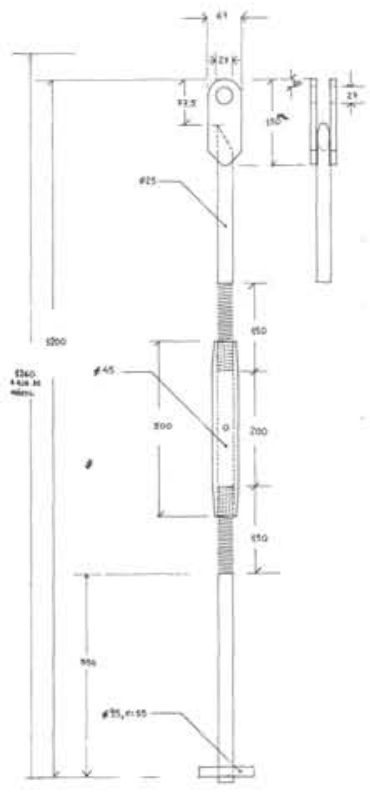

Tensor del capuchon supenor

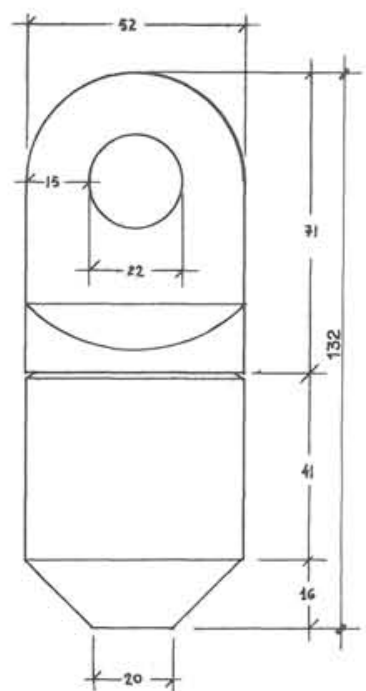

Terminal de cable a bulon. 


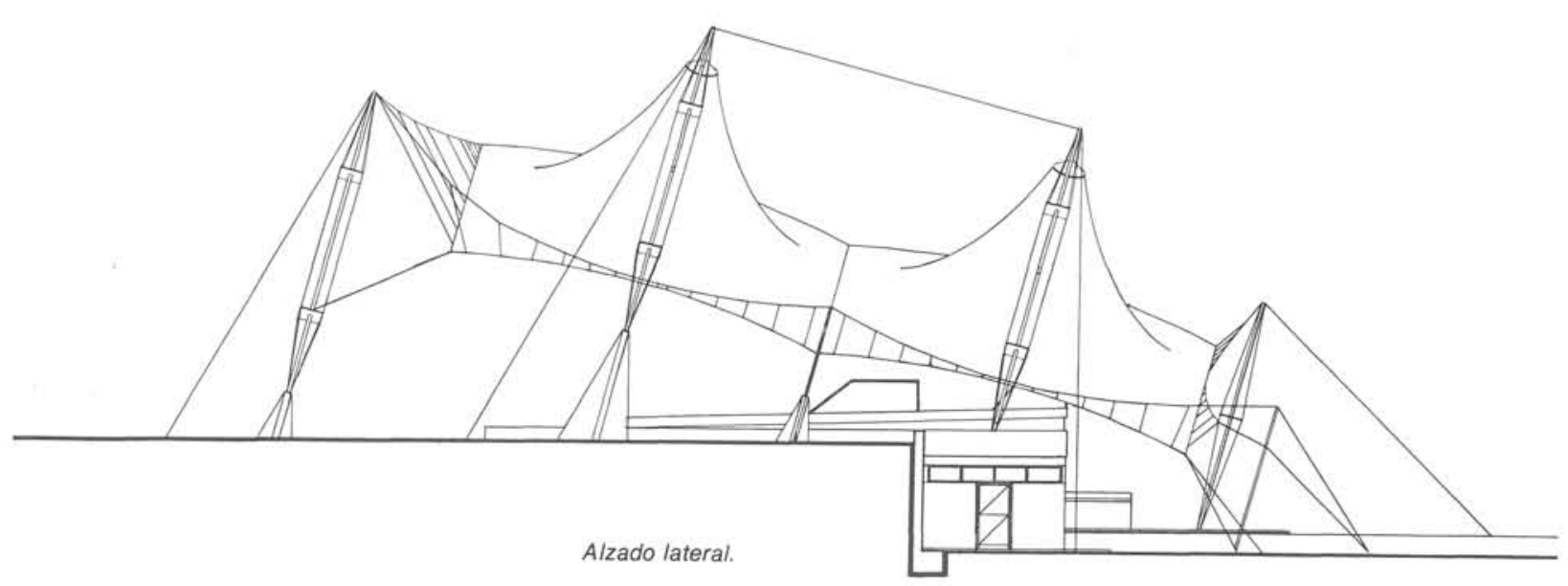

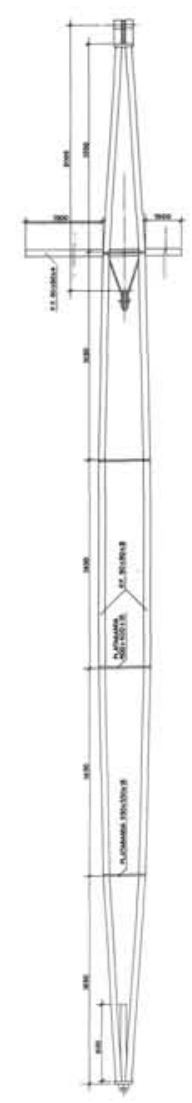

Mástil corto.
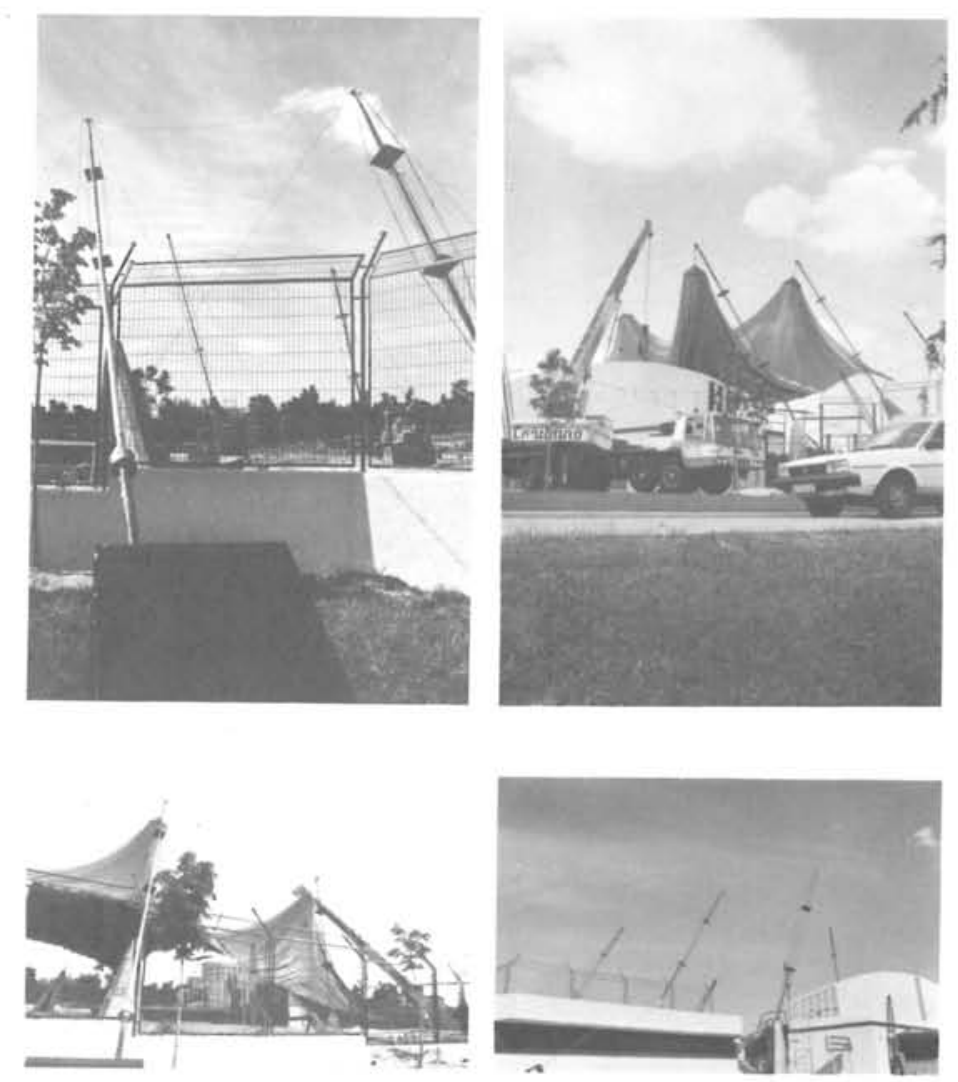

Fig. a
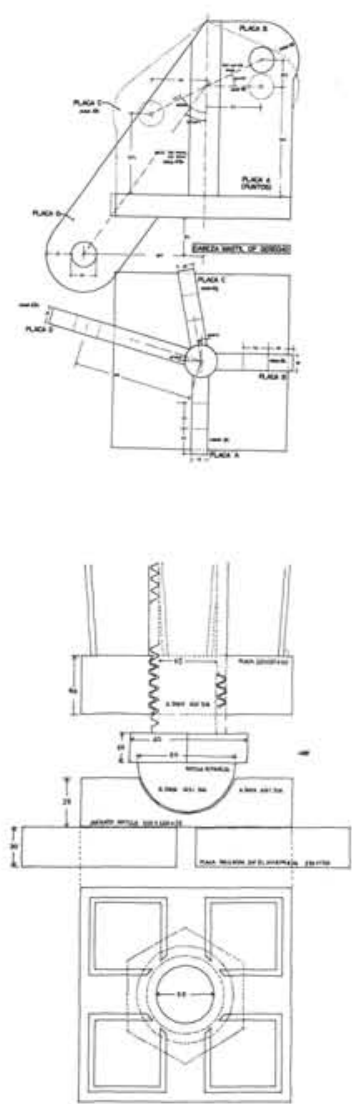

Cabeza y pie de mastil. alabeado, que exige el análisis de fuerzas en cada punto, según una dirección variable (Fig. 2).

Por otra parte, la isostaticidad de la estructura (apoyos y articulaciones) y su elasticidad, hace necesario un cuidadoso estudio de curvaturas en cada pieza de anclaje o empalme que permita "el giro" necesario según: su dirección y ajuste (tensores y tornillos), su plano axil (bulones y empalmes de borde) o su plano ortogonal (rótulas (figuras de la página anterior).
La mayoria de estas piezas se han resuelto por composición de otras que resolviendo las situaciones tipicas, solucionan las especificas: terminales de cables empalmados a: tensores, bulones, o enganches de borde. Para fijar la pieza terminal al cable se ha utilizado resina epoxi.

En cuanto a los mástiles -de considerable longitud: $11 \mathrm{~m}$ - se ha preferido emplear un perfil simple-tubo de $80 \times 80 \times 3 \mathrm{~mm}-$ con su longitud de pandeo acortada mediante tirantes en los tercios, con objeto de favorecer su ligereza, facili- 

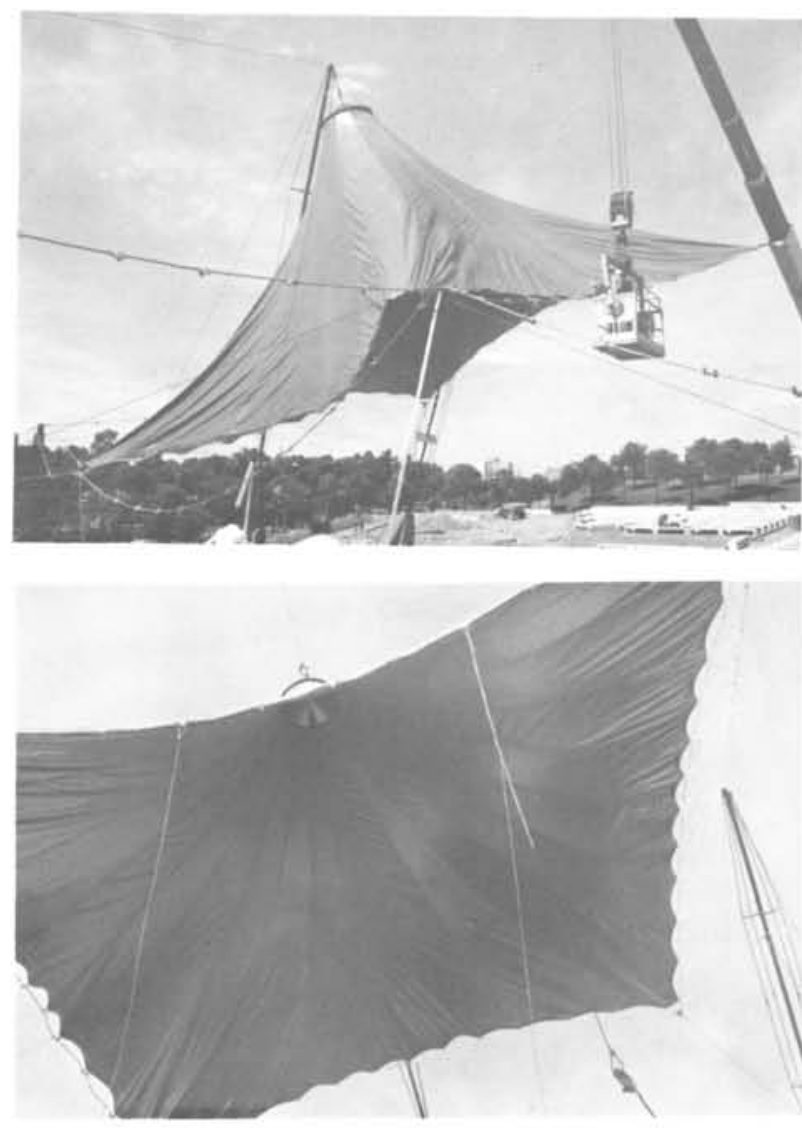

dad de manufactura y corrección, incluso en obra, frente a otros como antenas prefabricadas, etc.; cosa que, aunque dificil de suponer dado el grado de exactitud necesario para el replanteo de esta clase de obras (el funcionamiento mecánico de esta estructura se garantiza por la posición geométrica de sus puntos); sin embargo, es conveniente prever según las condiciones de producción en que nos movemos.

Mereceria mención especial el apartado dedicado a los empalmes y costuras de lona, tanto en la definición y casado de patrones de corte, como en el de refuerzos de borde, donde la importancia del cable como material de construcción se pone de manifiesto en estas estructuras escondido tras de cada vaina, solape, empalme... (Fig. 1).

El montaje, se realizó levantando primero los mástiles y fijando cada uno en su posición mediante tres cables (2 auxiliares), después se colgaron las catenarias y los arcos de borde correspondientes (una vez montados en el suelo) $y$, posteriormente, se fueron izando uno a uno los módulos siendo sucesivamente empalmados entre si.
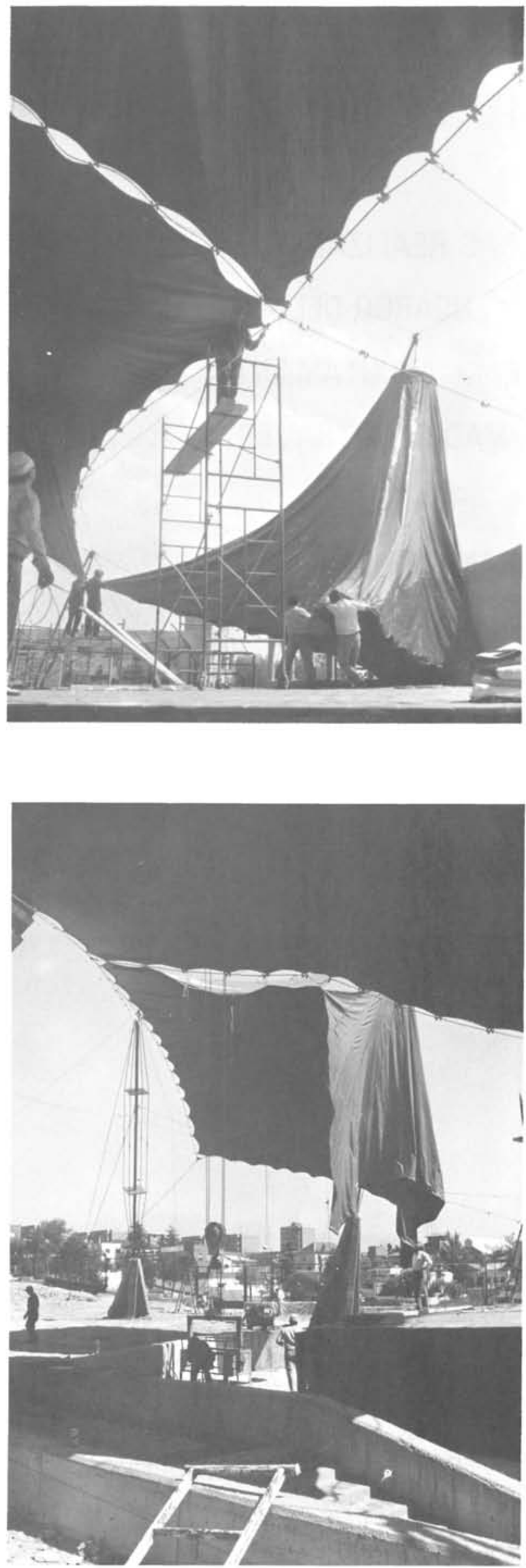